\title{
An Approximate Method for Computing Diffraction atterns Caused by Ionospheric Irregularities
}

\author{
Ralph Gagnon \\ Contribution From the Geo-Astrophysics Laboratory, Boeing Scientific Research Laboratories, \\ Seattle, Wash.
}

(Received October 21, 1963; revised December 13, 1963)

\begin{abstract}
A method, suitable for investigation of ionospheric phenomena, is derived for computing the amplitude and phase characteristics of an electromagnetic wave after it has been perturbed by a phase-distorting screen. Kirchhoff's integral for diffraction is used to evaluate the Fresnel zone fields in terms of a field distribution which is assumed to exist over a plane surface just inside the screen. The perturbing screen is assumed to distort the front of constant phase in one dimension only. By approximating the actual curved wave front with plane wave segments, and with the use of the approximations normally used in diffraction pattern analysis, the fields are evaluated in terms of a sum involving the Fresnel sine and cosine integrals. Several examples are given.
\end{abstract}

\section{Introduction}

Irregularities in the ionosphere have been the subject of considerable investigation. At lower frequencies, the existence of non-uniformities has been known for many years by the manifestations on ionogram records. More recently, it was by means of the same sounding: techniques that the first observations of large-scale moving irregularities were made by Munro $[1950,1958]$, who was able to study the time and spatial variations of the irregularities by means of spaced stations. Apparent tilts in the ionospheric layers were observed by Bramley [1953] using direction-finding techniques. In both these investigations, the scale of the irregularities was of the order of $100 \mathrm{~km}$. Another example of anomalous behavior is the sbort-lived increases in the amplitude of $F$-region reflections, which might be attributed to focusing [Whitehead, 1956].

At frequencies above the range covered by ionogram records, the ionosphere can be studied by means of its transmission properties as observed by the perturbations on extraterrestrial radio waves and satellite signals. Scattering, absorption, focusing, and diffraction are some of the anomalous effects one might expect to observe.

Small-scale diffraction effects of a statistical nature are particularly well suited to analytic investigation. Booker, Ratcliffe, and Shinn [1950] showed bow to relate the statistics of the wave which emerges from the diffracting screen to the statistics of the wave which reaches the ground, and this work has been extended by Hewish [1951], Fejer [1953], and others.

Transmission effects caused by large-scale irregularities are not so amenable to analysis, although they are observed experimentally. Tsuchiya and Morimoto [1960] and Fokker [1957] give evidence of anomalous scintillations of solar radio noise at meter wavelengths. Little and Lawrence [1960] have reported large-scale disturbances with dimensions of the order of $300 \mathrm{~km}$, observed by means of satellite signals.

It is felt that an analytic method to analyse these large-scale ionospheric diffraction and focusing effects is needed. This paper is concerned with the development of such a procedure.

\section{Analysis}

Let the electromagnetic field vector be denoted by $\mathbf{u}$. An observation point $\mathbf{x}$ lies within a source-free region enclosed by a surface $S$ comprising $S_{1}$ and $S_{2}$ (fig. 1). The variable point along the surface $S$ is denoted by $\mathbf{x}^{\prime}$. Then the relationship between the field at $\mathbf{x}$ and the 


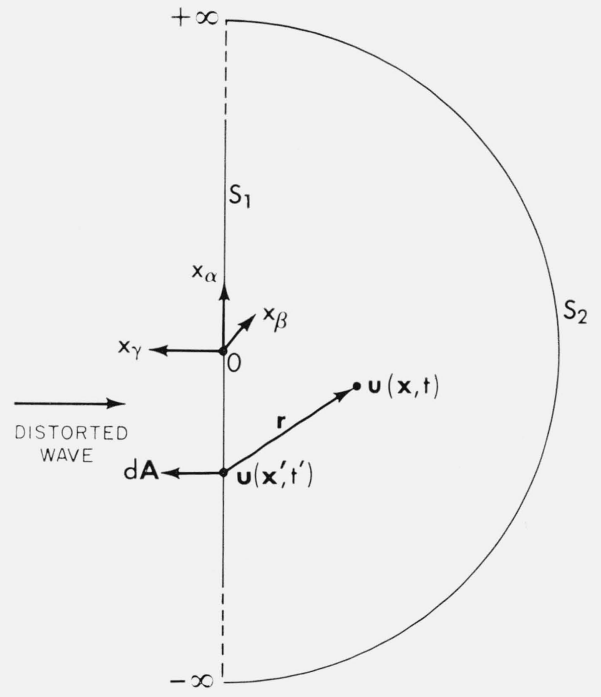

Figure 1. Geometry indicating surface of integration.

fields along $S\left(\mathbf{x}^{\prime}\right)$ can be expressed by the equation

$$
\mathbf{u}(\mathbf{x})=\frac{1}{4 \pi} \int \frac{e^{i k r}}{r}\left[\left(d \mathbf{A} \cdot \nabla^{\prime}\right) \mathbf{u}\left(\mathbf{x}^{\prime}\right)+\frac{i k \mathbf{u}\left(\mathbf{x}^{\prime}\right)}{r}(d \mathbf{A} \cdot \mathbf{r})\right],
$$

in the case where $r>\frac{c}{\omega}$. A harmonic time dependence $e^{-i \omega t}$ is assumed, $|\mathbf{k}|=\frac{\omega}{c}$, and $d \mathbf{A}$ is the outward vector differential of area along $S$. The integral over the surface $S_{2}$ can be assumed to vanish. Equation (1) is a form of the Fresnel-Kirchhoff diffraction integral [Jackson, 1962].

Let $x_{\alpha}^{\prime}$ and $x_{\beta}^{\prime}$ be two rectangular Cartesian coordinates on $S_{1}$, such that $d \mathbf{A}=d x_{\alpha}^{\prime} d x_{\beta}^{\prime} \mathbf{1}_{n}$ Let us suppose that the term $\mathbf{u}\left(\mathbf{x}^{\prime}\right)$ in (1) depends on one of these coordinates only, $x_{\alpha}^{\prime}$, and that on the plane $S_{1}$ the propagation vector $\mathbf{k}$ has an $x_{\alpha}$ component only. Let us further subdivide the integral into segments along $x_{\alpha}^{\prime}$. Thus

$$
\mathbf{u}_{j}(\mathbf{x})=\frac{1}{4 \pi} \int_{-\infty}^{+\infty} \int_{x_{\alpha j}}^{x_{\alpha j+1}} \frac{e^{i k r}}{r}\left[\left(\mathbf{1}_{n} \cdot \nabla^{\prime}\right) \mathbf{u}+i k \mathbf{u}\left(\mathbf{1}_{n} \cdot \frac{\mathbf{r}}{r}\right)\right] d x_{\alpha}^{\prime} d x_{\beta}^{\prime}
$$

where $x_{\beta}^{\prime}$ is the second coordinate and $\mathbf{1}_{n}$ is the outward unit normal. The total integral is given by the vector sum

$$
\mathbf{u}=\sum \mathbf{u}_{j}
$$

(where it is to be noted that the quantities $\mathbf{u}_{j}$ are in general complex).

Let us suppose that at any point on the surface $S_{1}$ the wave is a quasi-plane wave, with a vector amplitude $\mathbf{u}\left(\mathbf{x}^{\prime}\right)$, an angular frequency $\omega$, and a propagation vector $\mathbf{k}$ which is a slowly varying function of position. It will be assumed that the distortion is primarily phase distortion, and amplitude changes will be ignored, although these could be accounted for by allowing the magnitude of $\mathbf{u}\left(\mathbf{x}^{\prime}\right)$ on $S_{1}$ to vary from segment to segment.

If the segments are chosen small enough such that the propagation direction is constant over a segment, then the fields over that segment can be represented as

With this notation, (2) becomes

$$
\mathbf{u}\left(\mathbf{x}^{\prime}\right)=\mathbf{u}_{0 j} e^{i \mathbf{k}_{j} \cdot \mathbf{x}^{\prime}} .
$$

$$
\mathbf{u}_{j}(\mathbf{x})=\frac{i k \mathbf{u}_{0 j}}{4 \pi} \int_{-\infty}^{+\infty} \int_{x_{\alpha j}}^{x_{\alpha j+1}} \mathbf{1}_{n} \cdot\left[\frac{\mathbf{r}}{r}+\frac{\mathbf{k}_{j}}{k}\right] \frac{e^{i\left(k r+\mathbf{k}_{j} \cdot \mathbf{x}^{\prime}\right)}}{r} d x_{\alpha}^{\prime} d x_{\beta}^{\prime} .
$$




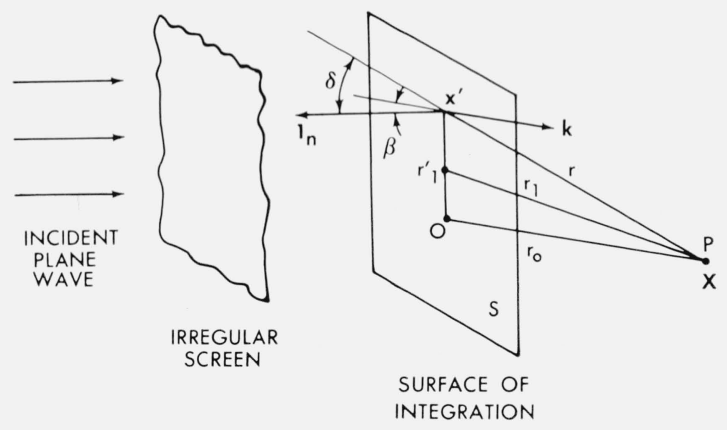

Figure 2. Diffraction at an irregular screen.

With the definition of the angles $\beta$ and $\delta$ as shown in figure 2, and with the assumption that the significant variation in the integrand occurs in the oscillation of the exponential term, (5) reduces to

$$
\mathbf{u}_{j}=-\frac{i k \mathbf{u}_{0 j}(\cos \beta+\cos \delta)}{4 \pi r_{1}} \int_{-\infty}^{+\infty} \int_{x_{\alpha j}}^{x_{\alpha j+1}} e^{i\left(k_{r}+\mathbf{k}_{j} \cdot \mathbf{x}^{\prime}\right)} d x_{\alpha}^{\prime} d x_{\beta}^{\prime},
$$

where $\delta$ and $r_{1}$ are evaluated at the point in the integrand where the angle between $\mathbf{k}$ and $\mathbf{r}$ is smallest.

Let the magnitude of the exponent of $(6)$ be called $F$, i.e.,

$$
F=k r+\mathbf{k} \cdot \mathbf{x}^{\prime},
$$

where the subscript is omitted. If $\mathbf{r}_{1}$ is restricted to be perpendicular to the $x_{\beta}^{\prime}$ axis, then terms up to second order in the Taylor series expansion of $F$ around $\mathbf{x}_{1}^{\prime}$ are

$$
F\left(\mathbf{x}^{\prime}\right)=a+b\left(\Delta x^{\prime}{ }_{\beta}+c\right)^{2}+d\left(\Delta x_{\alpha}^{\prime}+e\right)^{2},
$$

where $\mathbf{r}_{1}=\mathbf{x}-\mathbf{x}_{1}^{\prime}, \Delta \mathbf{x}^{\prime}=\mathbf{x}^{\prime}-\mathbf{x}_{1}^{\prime}, \mathbf{Q}=\frac{\mathbf{k}}{k}-\frac{\mathbf{r}_{1}}{r_{1}}$, and where

$$
\begin{aligned}
& a=k r_{1}+\mathbf{k} \cdot \mathbf{x}_{1}^{\prime}-\frac{k r_{1} Q_{\alpha}^{2}}{2 \cos ^{2} \delta}, \\
& b=\frac{k}{2 r_{1}} \\
& c=0 \\
& d=\frac{k \cos ^{2} \delta}{2 r_{1}} \\
& e=\frac{r_{1} Q_{\alpha}}{\cos ^{2} \delta} .
\end{aligned}
$$

The substitution of (8) into (6) results in an integral which can be evaluated in terms of the Fresnel sine and cosine integrals. The result is

where

$$
\mathbf{u}_{j}=-i k \mathbf{u}_{0 j} e^{i a} \frac{\cos \beta+\cos \delta}{8 r_{1}(b d)^{1 / 2}}\left[(1+i) C\left(y_{\alpha}^{\prime}\right)-(1-i) S\left(y_{\alpha}^{\prime}\right)\right]_{y_{\alpha j}^{\prime}}^{y_{\alpha j+1}^{\prime}}
$$

$$
y_{\alpha}^{\prime}=\left(\frac{2 d}{\pi}\right)^{1 / 2}\left(x_{\alpha}^{\prime}-x_{\alpha 1}^{\prime}+e\right),
$$


and $C(x)$ and $S(x)$ are the Fresnel sine and cosine integrals, defined by

$$
\begin{aligned}
& C(x)=\int_{0}^{x} \cos \left(\frac{\pi}{2} \tau^{2}\right) d \tau, \\
& S(x)=\int_{0}^{x} \sin \left(\frac{\pi}{2} \tau^{2}\right) d \tau .
\end{aligned}
$$

To obtain the desired solution, these $\mathbf{u}_{j}$ 's can then be substituted into (3).

In order to compute (9), $\mathbf{u}_{0 j}$ must be known. In general, the phase must be continuous in crossing from one segment to the next. Thus

$$
\mathbf{u}_{0 j} e^{i \mathbf{k}_{j} \cdot \mathbf{x}_{i}^{\prime}}=\mathbf{u}_{0 j+1} e^{i \mathbf{k}_{j+1} \cdot \mathbf{x}_{i}^{\prime}},
$$

where $\mathbf{x}_{j}^{\prime}$ is the point on $S$ where the $j$ th and the $j+1$ th segments meet, and $x_{\beta}^{\prime}=0$. Thus given $\mathbf{u}_{0 j}$, the phase of $\mathbf{u}_{0 j+1}$ is computed from the equation

$$
\mathbf{u}_{0 j+1}=\mathbf{u}_{0 j} e^{i\left(\mathbf{k}_{j}-\mathbf{k}_{i+1}\right) \cdot \mathbf{x}_{j}^{\prime}}
$$

On the other hand, it may be desired to introduce arbitrary "step" discontinuities in the phase. Then (12) can be replaced by

$$
\mathbf{u}_{0 j+1}=\mathbf{u}_{0 j} e^{i\left(\mathbf{k}_{j}-\mathbf{k}_{j+1}\right) \cdot \mathbf{x}_{j}^{\prime}+i \theta_{j+1}},
$$

where $\theta_{j+1}$ is the desired phase discontinuity in crossing from the $j$ th to the $j+1$ th segment.

Equation (13), which determines the vector coefficients, (9), which determines the contributions from each straight-line segment, and (3), which sums the various contributions, are the equations from which a large variety of diffraction-focusing patterns can be calculated.

\section{Some Computed Examples}

These equations have been incorporated into a computer program for the purpose of evaluating model diffraction patterns. These have been computed in a manner for comparison with the data from a particular lobe-sweep interferometer system [Lansinger and Gagnon, 1961]. This interferometer is located approximately along an east-west baseline. The data take the form of an amplitude plot, which is a measure of the product of the amplitudes at each of the two interferometer antennas, and a phase plot, which is a measure of the phase difference between the signals at the antennas.

Figure 3 is a curve showing the theoretical response of a system such as this to a sudden phase discontinuity. The parameters are

$\begin{array}{lrl}\text { Frequency } & 50 \mathrm{Mc} / \mathrm{s} . \\ \text { Baseline } & 200 \mathrm{~m} . \\ \text { Incident angle } & 45 \mathrm{deg} . \\ \text { Slant height }(r) & 420 \mathrm{~km} . \\ \text { Phase discontinuity } & 30 \mathrm{deg} .\end{array}$

The frequency and baseline chosen here for illustrative purpose are not the same as those of the interferometer in question, but are lower and longer, respectively. These parameters are easy to vary in the computations, and the values used here have been chosen as being of general interest. The independent variable "distance" on the curve is distance along the ground. The curve represents either the pattern as seen at a single point as the irregularity drifts by, or the variation in intensity over the ground at a particular instant of time. 


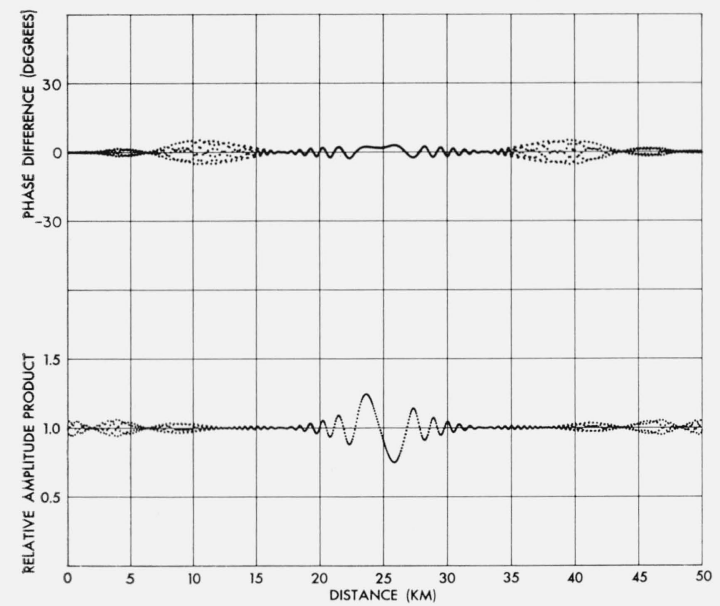

FiguRE 3. Diffraction pattern resulting from an ionospheric phase discontinuity.

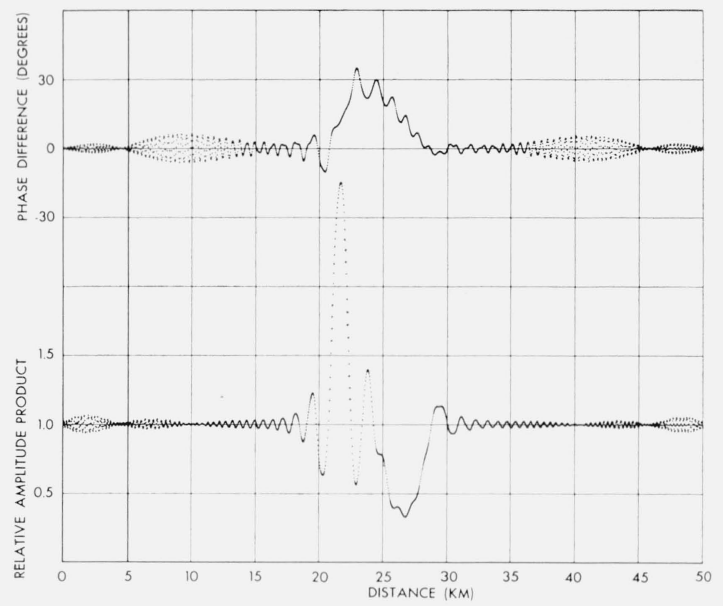

Figure 4. Diffraction pattern resulting from an ionospheric "wedge"

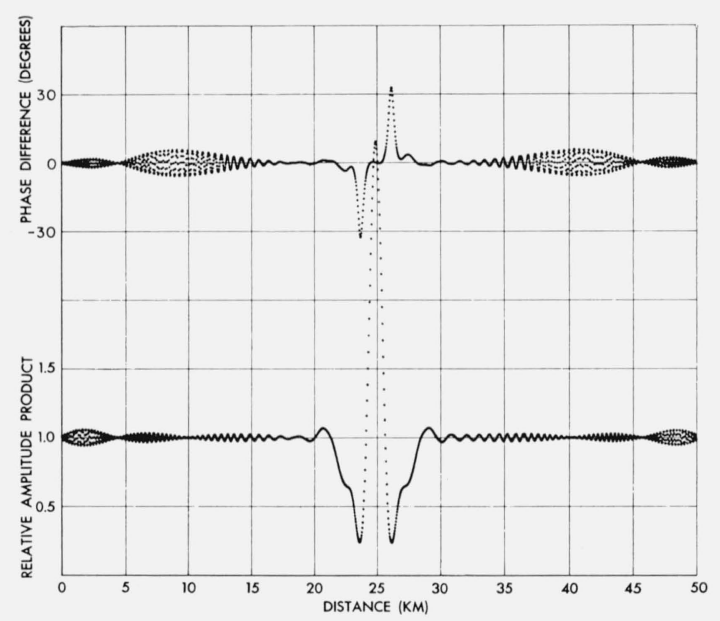

Figure 5. Diffraction pattern resulting from an ionospheric lens.

Figure 4 is an example of the pattern caused by a "wedge." The wedge is represented by a single segment over which the refraction is constant, where the term refraction refers to the angular deviation of the propagation vector from its undistorted orientation. The length of the wedge is $5 \mathrm{~km}$, and the refraction is $10 \mathrm{~min}$. The other parameters are the same as before.

Figure 5 is a plot for the pattern resulting from a simple "lens." An irregularity is simulated using a sine-wave distribution, with 71 equal-length segments which total $5 \mathrm{~km}$. The refraction of the $n$th segment is given by

$$
\text { refraction }=7 \sin (5 n) \text { minutes. }
$$

The refraction has been chosen to place the interferometer roughly at the focus of the lens.

This particular curve took 25 min of IBM 7094 computer time to compute. However, the same lens was approximated with only 15 equal-length segments with only a little difference between the two curves. This corresponds to a minimum segment length of a few hundred meters in this case. By also suitably reducing the point density, a time saving by a factor of about 15 is possible. Curves such as the wedge shown in figure 4 take typically 15 to 30 sec on production runs. 


\section{Summary and Conclusion}

A method has been shown for computing the ionospheric diffraction-refraction patterns resulting from an assumed field distribution in terms of tabulated functions. Although the method is too involved for hand calculations, it has been found convenient for computer applications.

Several examples have been shown. These include patterns resulting from a phase discontinuity, a wedge, and a lens. The patterns shown are not to be considered typical of the shapes of the irregularities causing them, as there is an almost endless variety of ground patterns which occur as one varies the parameters in a given problem.

Although one can compute a ground pattern from the phase-distorted wave which gives rise to it, one cannot conveniently deduce the phase-distorted wave from the ground pattern. Thus the primary application is for hypothesis testing. With an idea as to the nature of a particular disturbance, a way is provided to check upon and refine one's estimate. It is hoped that the method described here will prove to be a useful tool for this purpose.

The author acknowledges helpful discussions with H. E. Brandt, J. F. Kenney, and J. M. Lansinger. Much of the programming was done by M. D. Gray.

\section{References}

Booker, H. G., J. A. Ratcliffe, and D. H. Shinn (Sept. 1950), Diffraction from an irregular screen with applications to ionospheric problems, Phil. Trans. Roy. Soc. London A242, 579-607.

Bramley, E. N. (Oct. 1953), Direction finding studies of large-scale ionospheric irregularities, Proc. Roy. Soc. (London) A220, 39-61.

Fejer, J. A. (Dec. 1953), The diffraction of waves in passing through an irregular refracting medium, Proc. Roy. Soc. (London) A220, 455-471.

Fokker, A. D. (1957), A peculiar type of scintillation of solar radio radiation, I.A.U. Symposium No. 4: Radio Astronomy, p. 371 (Cambridge University Press).

Hewish, A. (Oct. 1951), The diffraction of radio waves in passing through a phase-changing ionosphere, Proc. Roy. Soc. (London) A209, 81-96.

Jackson, J. D. (1962), Classical electrodynamics, p. 283 (John Wiley and Sons, Inc., New York, N.Y.).

Lansinger, J. M., and R. Gagnon (1961), Boeing lobe-sweep interferometer system, Boeing document D1-82-0122.

Little, C. G., and R. S. Lawrence (Jan 1960), The use of polarization fading of satellite signals to study the electron content and irregularities in the ionosphere, J Res. NBS 64D (Radio Prop.), No. 4, $335-346$.

Munro, G. H. (July 1950), Travelling disturbances in the ionosphere, Proc. Roy. Soc. (London) A202, $208-223$.

Munro, G. H. (March 1958), Travelling ionospheric disturbances in the $F$ region, Australian J. Phys. 11, 91-112.

Tsuchiya, A., and M. Morimoto (1960), Anomalous scintillation of solar radio emission, Rep. Ionosphere Res. Japan 14, No. 4, 450-452.

Whitehead, J. D. (1956), The focusing of short radio waves reflected from the ionosphere, J. Atmospheric Terrest. Phys. 9, 269-275.

(Paper 68D6-371) 